\title{
A Continuous Characterization of Triebel-Lizorkin Spaces Associated with Hermite Expansions
}

\author{
Shuli Gong, ${ }^{1}$ Bolin $\mathrm{Ma}^{2}$ and Zunwei $\mathrm{Fu}^{1}$ \\ ${ }^{1}$ Department of Mathematics, Linyi University, Linyi, Shandong 276005, China \\ ${ }^{2}$ College of Mathematics Physics and Information Engineering, Jiaxing University, Jiaxing, Zhejiang 314001, China \\ Correspondence should be addressed to Zunwei Fu; zwfu@mail.bnu.edu.cn
}

Received 27 January 2015; Accepted 28 April 2015

Academic Editor: David R. Larson

Copyright (c) 2015 Shuli Gong et al. This is an open access article distributed under the Creative Commons Attribution License, which permits unrestricted use, distribution, and reproduction in any medium, provided the original work is properly cited.

We study the properties of the Triebel-Lizorkin spaces associated with the multidimensional Hermite expansions on $\mathbb{R}^{d}(d \geq 1)$. In addition to the endpoint estimates, we give the continuous characterizations of these spaces via harmonic and thermic extensions from $\mathbb{R}^{d}$ into $\mathbb{R}_{+}^{d+1}$. Based on this result, we obtain the boundedness of Riesz transform associated with the Hermite expansions.

\section{Introduction}

For a smooth functional series $\left\{\varphi_{j}\right\}_{-\infty}^{\infty}$, if it satisfies the following conditions:

(i) $\operatorname{supp} \varphi_{j} \subset\left\{x: 2^{j-1} \leq|x| \leq 2^{j+1}\right\}, j=1,2,3, \ldots$,

(ii) $2^{j k}\left|D^{(k)} \varphi_{j}(x)\right| \leq C_{k}, j \in Z, k \in \mathbb{N}_{0}$,

(iii) $\sum_{j=-\infty}^{\infty} \varphi_{j}(x)=1, x \in \mathbb{R}^{d}$,

then we call $\left\{\varphi_{j}\right\}_{-\infty}^{\infty}$ a smooth dyadic resolution of unity in $\mathbb{R}^{d}(d \geq 1)$. As proved in [1], it is easy to construct such a resolution of unity.

Let $D$ be a self-adjoint operator acting on $L^{2}\left(\mathbb{R}^{d}\right)$, and let $d E$ be its spectral resolution which is defined by

$$
D f=\int_{0}^{\infty} \lambda d E(\lambda) f .
$$

For $\alpha \in \mathbb{R}, 0<p<\infty, 0<q \leq \infty$, and a $C^{\infty}$ function $\varphi \in\left\{\varphi_{j}\right\}_{-\infty}^{\infty}$, we define the Triebel-Lizorkin norm associated with $D$ by

$$
\|f\|_{F_{p}^{\alpha, q}(D)}=\left\|\left(\sum_{j=-\infty}^{\infty}\left(2^{j \alpha}\left|\varphi_{j}(D) f(x)\right|\right)^{q}\right)^{1 / q}\right\|_{L^{p}\left(\mathbb{R}^{d}\right)},
$$

where

$$
\varphi_{j}(D) f=\varphi\left(2^{-j} D\right) f=\int_{0}^{\infty} \varphi\left(2^{-j} \lambda\right) d E(\lambda) f .
$$

Note that if $D=\Delta=-\sum_{j=1}^{d}\left(\partial^{2} / \partial x_{j}^{2}\right)$ is the Laplacian on $\mathbb{R}^{d}$, then the norm $\|f\|_{F_{p}^{\alpha, q}(D)}$ in (2) is equivalent to the classical Triebel-Lizorkin norm $\|f\|_{F_{p}^{\alpha, q}(\Delta)}$; see [2]. The main goal of this paper is to study the Triebel-Lizorkin space $F_{p}^{\alpha, q}(D)$ when $D$ is the Hermite operator.

The $d$-dimensional Hermite functions on the $d$ dimensional Euclidean space $\mathbb{R}^{d}(d \geq 1)$ are defined by

$$
h_{\alpha}(x)=h_{\alpha_{1}}\left(x_{1}\right) \times h_{\alpha_{2}}\left(x_{2}\right) \times \cdots h_{\alpha_{d}}\left(x_{d}\right),
$$

where $\alpha=\left(\alpha_{1}, \ldots, \alpha_{d}\right), \alpha_{i} \in\{0,1, \ldots\}, x=\left(x_{1}, \ldots, x_{d}\right)$, and

$$
h_{k}(s)=\left(\pi^{1 / 2} 2^{k} k !\right)^{-1 / 2} H_{k}(s) \exp \left(-\frac{s^{2}}{2}\right) \text {, }
$$

$$
k=0,1, \ldots,
$$

are the one-dimensional Hermite functions; $H_{k}(s)$ denotes the $k$ th Hermite polynomial. We know that the Hermite functions form an orthonormal basis for $L^{2}\left(\mathbb{R}^{d}\right)$ and that $h_{\alpha}$ 
are eigenfunctions of the Hermite operator $H=-\Delta+|x|^{2}$; that is,

$$
H h_{\alpha}=(2|\alpha|+d) h_{\alpha},
$$

where $|\alpha|=\alpha_{1}+\cdots+\alpha_{d}$. Moreover, the operator $H$ is positive and symmetric in $L^{2}$ on the domain $C_{c}^{\infty}\left(\mathbb{R}^{d}\right)$. It is easy to check that the operator $H$ given by

$$
H\left(\sum\left\langle f, h_{\alpha}\right\rangle h_{\alpha}\right)=\sum(2|\alpha|+d)\left\langle f, h_{\alpha}\right\rangle h_{\alpha}
$$

is a self-adjoint extension of $H$ on the domain

$$
\begin{aligned}
& \operatorname{Dom}(H) \\
& =\left\{f \in L^{2}\left(\mathbb{R}^{d}\right): \sum\left|(2|\alpha|+d)\left\langle f, h_{\alpha}\right\rangle\right|^{2}<\infty\right\} .
\end{aligned}
$$

The operator $H$ has the discrete spectrum $\{2 k+d: k=$ $0,1, \ldots\}$ and admits the spectral decomposition

$$
H f=\sum_{k=0}^{\infty}(2 k+d) P_{k} f, \quad f \in \operatorname{Dom}(H),
$$

where $P_{k}$ is a spectral projection that is defined to be $P_{k} f=$ $\sum_{|\alpha|=k}\left\langle f, h_{\alpha}\right\rangle h_{\alpha}$. As to the other properties of the Hermite functions, the interested readers can refer to Thangavelu's monograph [3] for a detailed description.

In the past few years, many authors have studied the Triebel-Lizorkin spaces associated with the Hermite operators. In [4], Epperson studied the Triebel-Lizorkin spaces associated with the one-dimensional Hermite operators for

$$
\operatorname{supp} \varphi \subset\left[\frac{1}{2}, 2\right], \quad|\varphi(\lambda)|>c>0, \lambda \in\left[\frac{3}{4}, \frac{7}{4}\right] .
$$

Using Mehler's formula, Epperson proved that the definition of the corresponding space $\|f\|_{F_{p}^{\alpha, q}(H)}$ is independent of the particular choice of the function $\varphi$. In [5], Dziubański continued this study and proved that the results in [4] also hold for the multidimensional Hermite expansions. Besides, Petrushev and $\mathrm{Xu}$ [6] showed that the Triebel-Lizorkin on $\mathbb{R}^{d}$ induced by Hermite expansions can be characterized in terms of the needlet coefficients and that the Hermite-TriebelLizorkin space is, in general, different from the respective classical spaces. In addition, Olafsson and Zheng [7] study the Triebel-Lizorkin space associated with the Peetre type maximal function for Hermite operator $H$, which is defined by

$$
\varphi_{j, s}^{*} f(x)=\sup _{y \in \mathbb{R}^{d}} \frac{\left|\varphi_{j}(H) f(y)\right|}{\left(1+2^{j / 2|x-y|}\right)^{s}}, \quad j \in \mathbb{Z}, s>0 .
$$

They gave the maximal characterization of this space as follows.

Theorem GS. Let $\varphi_{j} \in\left\{\varphi_{j}(x)\right\}_{-\infty}^{\infty}, 0<p<\infty, 0<q \leq \infty$, and $\alpha \in \mathbb{R}$. If $s>d / \min (p, q)$, then

$$
\|f\|_{F_{p}^{\alpha, q}} \sim\left\|\left\{2^{j \alpha} \varphi_{j, s}^{*}(H) f\right\}\right\|_{L^{p}\left(l^{q}\right)},
$$

where $\varphi_{j, s}^{*}(H) f$ is the Peetre type maximal function for Hermite operator.

In [3], Thangavelu introduced Riesz transforms associated with Hermite expansions and proved that they are bounded operators on $L^{p}\left(\mathbb{R}^{d}\right), 1<p<\infty$. In [8], Harboure et al. gave a different proof to show that the $L^{p_{-}}$ norms of these operators are bounded by a constant not depending on the dimension $d$. Moreover, they also defined the Riesz transforms of higher order and obtained the free dimensional estimates of the $L^{p}$-bounds of these operators. After that, Stempak and Torrea [9] obtained the weighted $L^{p}$-inequalities for the gradient square function associated with the Poisson semigroup. They get the result proposed in [3] by using a slightly different proof and they also get the analogous result for the $g$-function associated with the Poisson semigroup.

Although many results have been obtained about the Triebel-Lizorkin spaces associated with the Hermite operators, all of them only discuss the case of the discrete quasinorm of the Triebel-Lizorkin spaces associated with the Hermite operators. Up to now, as we know, there are few papers related to the continuous case due to the fact that the Hermite expansions do not satisfy the general convolution property. This makes the study of the continuous case a meaningful problem. We first present a general characterization of the Triebel-Lizorkin spaces associated with the multidimensional Hermite expansions satisfied continuous quasi-norm on $\mathbb{R}^{d}$. Furthermore, analogous to the classical Triebel-Lizorkin space, we construct the Littlewood-Paley theorem on these spaces. Finally, we obtain the endpoint estimate of these spaces and establish the boundedness of the Riesz transforms associated with Hermite functions expansions.

Throughout this paper, $|x|$ is the Euclidean norm of $x$ for $x \in \mathbb{R}^{d} ; A \sim B$ means $C_{1} A \leq B \leq C_{2} A$, where $C_{1}$, $C_{2}$ are positive constants; $C$ denotes a positive constant that may vary at every occurrence. For simplicity, we abbreviate $\|f\|_{F_{p}^{\alpha, q}(H)}$ to $\|f\|_{F_{p}^{\alpha, q}}$.

\section{The Characterizations of $F_{p}^{\alpha, q}$}

First, we give the definition of the Triebel-Lizorkin spaces associated with Hermite operators. Let $\varphi_{j} \in\left\{\varphi_{j}(x)\right\}_{-\infty}^{\infty}$, $\varphi_{j}(x)=\varphi\left(2^{-j} x\right)$; the Triebel-Lizorkin spaces associated with Hermite operator $H$, denoted by $F_{p}^{\alpha, q}, \alpha \in \mathbb{R}, 0<p$, and $q<$ $\infty$, are defined by the quasi-norm

$$
\|f\|_{F_{p}^{\alpha, q}}=\left\|\left(\sum_{j=-\infty}^{\infty}\left(2^{j \alpha}\left|\varphi_{j}(H) f(x)\right|\right)^{q}\right)^{1 / q}\right\|_{L^{p}\left(\mathbb{R}^{d}\right)},
$$

where

$$
\varphi_{j}(H) f(x)=\sum_{k=0}^{\infty} \varphi\left(2^{-j}(2 k+d)\right) P_{k} f(x) .
$$

The results of Dziubański [5] show that the definition of $F_{p}^{\alpha, q}$ does not depend on the particular choice of $\varphi$. Next, we will 
give the general characterization of Triebel-Lizorkin spaces associated with the Hermite expansions.

Theorem 1. Let $\alpha \in \mathbb{R}, 1<p, q<\infty$, and $\varphi \in S\left(\mathbb{R}^{d}\right)$; let $\varphi_{j}(x)=\varphi\left(2^{-j} x\right)$. If $x \in \mathbb{R}^{d} \backslash\{0\}$ and $j \in \mathbb{Z}$, then

$$
\left\|\left(\int_{0}^{\infty}\left|t^{-\alpha} \varphi(t H) f(\cdot)\right|^{q} \frac{d t}{t}\right)^{1 / q}\right\|_{L^{p}\left(\mathbb{R}^{d}\right)}
$$

(with usual modification when $q=\infty$ ) is an equivalent quasinorm in $F_{p}^{\alpha, q}$.

This theorem shows that any "discrete" quasi-norm of the Triebel-Lizorkin spaces associated with the Hermite expansions of type (13) has a "continuous" counterpart (15).

In order to prove our theorem, we need some necessary lemmas.

Lemma 2. Let $\left\{\varepsilon_{k}\right\},\left\{v_{k}\right\}$ be two sequences of complex numbers. Then, for any $l$, we have

$$
\sum_{k} \varepsilon_{k} v_{k}=\sum_{k}\left(\Delta^{l} \varepsilon_{k}\right) A_{k}^{l-1} S_{k}^{l-1}
$$

where

$$
S_{k}^{l-1}=\left(A_{k}^{l-1}\right)^{-1} \sum_{i=1}^{k} A_{k-i}^{l-1} v_{i}
$$

is the $(l-1)$ th linear mean of first $k$ terms of $\left\{v_{k}\right\}$ and $\Delta^{m}$ is the $m$ th difference operator.

Since the proof of Lemma 2 is easy, we omit the proof for the sake of compactness of the paper.

In [3], a basic estimate for the kernel of Riesz means for the Hermite expansions has been obtained. We describe the result in the following lemma.

Lemma 3. For $\alpha>(3 d-2) / 6$, the following estimate is valid:

$$
\begin{aligned}
& \left|S_{R}^{\alpha}(x, y)\right| \leq C R^{d / 2}\left\{\left(1+R^{1 / 2}|x-y|\right)^{-\alpha+(3 d-2) / 6-1}\right. \\
& \left.\quad+\left(1+R^{1 / 2}|x+y|\right)^{-\alpha+(3 d-2) / 6-1}\right\}
\end{aligned}
$$

where $S_{R}^{\alpha}(x, y)$ is the kernel of the Riesz means $S_{R}^{\alpha} f$ for the Hermite expansions, which is defined by

$$
S_{R}^{\alpha} f=\sum_{k=0}^{\infty}\left(1-\frac{2 k+d}{R}\right)_{+}^{\alpha} P_{k} f .
$$

Lemma 4. Assume that $f \in L^{p}\left(\mathbb{R}^{d}\right), 1 \leq p<\infty$, and $\alpha>$ $(9 d-8) / 6$. We have the pointwise inequality

$$
S_{*}^{\alpha} f(x) \leq C M_{p} f(x),
$$

where

$$
S_{*}^{\alpha} f(x)=\sup _{R>0}\left|S_{R}^{\alpha} f(x)\right|
$$

is the maximal operator and

$$
M_{p} f(x)=\left(\sup _{r>0} \frac{1}{B_{r}(x)} \int_{B_{r}(x)}|f(x)|^{p} d y\right)^{1 / p}
$$

is the Hardy-Littlewood maximal function of $|f|^{p}$ raised to the power $1 / p$ and $B_{r}(x)$ is the ball of radius $r$ centered at $x$.

Proof. The proof is based on the estimate of Lemma 3:

$$
\begin{aligned}
& \left|S_{R}^{\alpha} f(x)\right|=\left|\int_{\mathbb{R}^{n}} S_{R}^{\alpha}(x, y) f(y) d y\right| \\
& \quad \leq\left(\int_{\mathbb{R}^{d}}\left|S_{R}^{\alpha}(x, y)\right|^{q}\right)^{1 / q}\left(|f(y)|^{p}\right)^{1 / p} \\
& \quad \leq C\left(\int_{\mathbb{R}^{d}}\left|R^{d / 2}\left(1+R^{1 / 2}|x-y|\right)^{-\alpha+(3 d-2) / 6-1}\right|^{q} d y\right)^{1 / q} \\
& \quad\|f\|_{L^{p}} \\
& \quad+C\left(\int_{\mathbb{R}^{d}}\left|R^{d / 2}\left(1+R^{1 / 2}|x+y|\right)^{-\alpha+(3 d-2) / 6-1}\right|^{q} d y\right)^{1 / q} \\
& \quad \cdot\|f\|_{L^{p}}=I+I I,
\end{aligned}
$$

where $1 / p+1 / q=1$. Consider a partition of $\mathbb{R}^{d}$ into dyadic annuli $B_{j}=\left\{y: 2^{j} \leq|x-y| \leq 2^{j+1}\right\}$. For a given function $f$, we set $f_{j}(y)=f(y)$ for $2^{j} \leq|x-y| \leq 2^{j+1}$ and $f_{j}(y)=0$ otherwise. Then

$$
\begin{aligned}
I \leq & C \sum_{j=-\infty}^{\infty}\left(\int_{B_{j}}\left|R^{n / 2}\left(1+R^{1 / 2}|x-y|\right)^{-\alpha+(3 d-2) / 6-1}\right|^{q} d y\right)^{1 / q} \\
& \cdot\left\|f_{j}\right\|_{L^{p}} \\
& \leq C \sum_{j=-\infty}^{\infty} R^{d / 2}\left(1+R^{1 / 2} 2^{j}\right)^{-\alpha+(3 d-2) / 6-1}\left|B_{j}\right|^{1 / q+1 / p} M_{p} f(x) \\
& \leq C \sum_{j=-\infty}^{\infty}\left(R^{1 / 2} 2^{j}\right)^{d}\left(1+R^{1 / 2} 2^{j}\right)^{-\alpha+(3 d-2) / 6-1} M_{p} f(x) \\
& =G\left(R^{1 / 2}\right),
\end{aligned}
$$

where

$$
G(t)=\sum_{j=-\infty}^{\infty}\left(t 2^{j}\right)^{d}\left(1+t 2^{j}\right)^{-\alpha+(3 d-2) / 6-1} .
$$

It is obvious that $G(t)$ is locally bounded. Since $G\left(2^{i} t\right)=$ $G(t)$, it is bounded on $(0, \infty)$ (see [3]). The boundedness of II can also be obtained by replacing $y$ in the proof of the boundedness of I by $-y$ and following from the same estimate tactics as above. Hence, we have $S_{*}^{\alpha} f(x) \leq C M_{p} f(x)$.

We give the proof of Theorem 1 in what follows.

Proof. Consider the following.

Step 1. Let $f \in F_{p}^{\alpha, q}$. We will prove that $\|f\|_{F_{p}^{\alpha, q}}$ in (13) can be estimated from above by the quasi-norm in (15). Let 
$\rho_{j} \in\left\{\varphi_{j}(x)\right\}_{-\infty}^{\infty}$ and $l-1>(9 d-8) / 6$. It follows from Lemma 4 that

$$
\begin{aligned}
& \left|\rho_{j}(H) f(x)\right|=\left|\frac{\rho_{j}(H)}{\varphi(\lambda H)} \varphi(\lambda H) f(x)\right| \\
& =\mid \sum_{k} \Delta^{l}\left(\frac{\rho_{j}(2 k+d)}{\varphi(\lambda(2 k+d))}\right) A_{k}^{l-1} S_{k}^{l-1}\left(\varphi_{j}(\lambda H) f\right) \\
& \cdot(x)\left|\leq \sum_{k}\right| \Delta^{l}\left(\frac{\rho_{j}(2 k+d)}{\varphi(\lambda(2 k+d))}\right) A_{k}^{l-1} \mid \\
& \cdot\left|M\left(\varphi_{j}(\lambda H) f\right)(x)\right| .
\end{aligned}
$$

For the first term of the right-hand side of (26), taking the supremum with respect to $\lambda$ on $1<\lambda<2$ yields

$$
\begin{aligned}
& \sup _{1 \leq \lambda \leq 2} \sum_{k}\left|\Delta^{l}\left(\frac{\rho_{j}(2 k+d)}{\varphi_{j}(\lambda(2 k+d))}\right) A_{k}^{l-1}\right| \\
& \leq C \sum_{k} \mid 2^{(-j+1) l} \sum_{i=0}^{l} C_{l}^{i} \rho^{(l-i)}\left(2^{-j}(2 k+d)\right) \\
& \cdot\left(\frac{1}{\varphi\left(2^{-j}(2 k+d)\right)}\right)^{(i)} \mid k^{l-1} \leq C \sum_{k} 2^{2(-j+1) l} \\
& \cdot \frac{\left(1+\left|2^{-j}(2 k+d)\right|\right)^{b}}{\left(1+\left|2^{-j}(2 k+d)\right|\right)^{a}} k^{l-1} \leq C \sum_{k} 2^{2(-j+1) l} 2^{(a-b) j} \\
& \cdot \frac{1}{(1+|2 k+d|)^{a-b}} k^{l-1} .
\end{aligned}
$$

We choose $a-b=2 l>l-1>0$; then we have

$$
\sup _{1 \leq \lambda \leq 2} \sum_{k}\left|\Delta^{l}\left(\frac{\rho_{j}(2 k+d)}{\varphi(\lambda(2 k+d))}\right) A_{k}^{l-1}\right| \leq C .
$$

Afterwards, integrating the modified inequality with respect to $\lambda$ that appears now only in the maximal function yields

$$
\left|\rho_{j}(H) f(x)\right| \leq C\left|\int_{1}^{2} M\left(\varphi_{j}(\lambda H) f\right)(x) d \lambda\right|
$$

where $M(f)$ is the Hardy-Littlewood maximal function.

For $1<p<\infty$ and $1<q<\infty$, we first multiply (29) by $2^{j \alpha}$; and then we apply the $l_{q}$-norm with respect to $j$; afterwards, we apply the $L^{p}$-norm with respect to $x$. That gives

$$
\begin{aligned}
& \left\|\left(\sum_{j=-\infty}^{\infty}\left(2^{j \alpha}\left|\rho_{j}(H) f(x)\right|\right)^{q}\right)^{1 / q}\right\|_{L^{p}} \\
& \leq C\left\|\left(\sum_{j=-\infty}^{\infty}\left|\int_{1}^{2} M\left(2^{\alpha j} \varphi_{j}(\lambda H) f\right)(x) d \lambda\right|^{q}\right)^{1 / q}\right\|_{L^{p}} \\
& \leq C\left\|\left(\sum_{j=-\infty}^{\infty} 2^{\alpha q j}\left|\int_{1}^{2} \sum_{k} \varphi\left(2^{-j} \lambda(2 k+d)\right) P_{k} f(x) d \lambda\right|^{q}\right)^{1 / q}\right\|_{L^{p}} \\
& \leq C\left\|\left(\sum_{j=-\infty}^{\infty} \int_{2^{-j}}^{2^{-j+1}}\left|t^{-\alpha} \sum_{k} \varphi(t(2 k+d)) P_{k} f(x)\right|^{q} \frac{d t}{t}\right)^{1 / q}\right\|_{L^{p}} \\
& \leq C\left\|\left(\int_{0}^{\infty}\left|t^{-\alpha} \sum_{k} \varphi(t(2 k+d)) P_{k} f(x)\right|^{q} \frac{d t}{t}\right)^{1 / q}\right\| \\
& \leq C\left\|\left(\int_{0}^{\infty}\left|t^{-\alpha} \varphi(t H) f(x)\right|^{q} \frac{d t}{t}\right)^{1 / q}\right\|
\end{aligned}
$$

Step 2. We will show that the quasi-norm in (15) can be

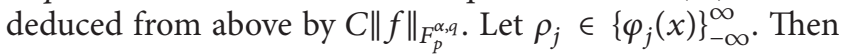
we have (13) with $\rho$ instead of $\varphi$. Let $\rho_{m}(x)=0,-m \in \mathbb{N}$. For $j \in \mathbb{Z}$, we get

$$
\begin{aligned}
2^{\alpha j} & \left(\varphi_{j}(H) f\right)(x) \\
& =\sum_{m=-\infty}^{\infty} 2^{\alpha j}\left(\varphi_{j}(H) \rho_{m+j}(H) f\right)(x) \\
& =\sum_{m=-\infty}^{K} \cdots+\sum_{m=K+1}^{\infty} \cdots
\end{aligned}
$$

We give the estimate of the first sum in (31). Let

$$
\widetilde{\rho}_{j}(x)=\left|2^{-j} x\right|^{\alpha_{1}} \rho_{j}(x) \quad j \in \mathbb{Z}, \alpha_{1}>\alpha .
$$

Then we have

$$
\begin{aligned}
& \sum_{m=-\infty}^{K} 2^{\alpha j}\left(\varphi_{j}(H) \rho_{m+j}(H) f\right)(x)=\sum_{m=-\infty}^{K} 2^{\alpha j} \\
& \cdot \sum_{k} \varphi\left(2^{-j}(2 k+d)\right) \rho_{m+j}(2 k+d) P_{k} f(x) \\
& =\sum_{m=-\infty}^{K} 2^{\alpha j} \sum_{k} \frac{\varphi\left(2^{-j}(2 k+d)\right)}{\left|2^{-(m+j)}(2 k+d)\right|^{\alpha_{1}} \widetilde{\rho}_{m+j}(2 k+d)} \\
& \cdot P_{k} f(x)=\sum_{m=-\infty}^{K} 2^{\alpha(j+m)} 2^{\left(\alpha_{1}-\alpha\right) m} \\
& \cdot \sum_{k}\left(\Delta^{l} \frac{\varphi\left(2^{-j}(2 k+d)\right)}{\left|2^{-j}(2 k+d)\right|^{\alpha_{1}}}\right) A_{k}^{l-1} S_{k}^{l-1}\left(\tilde{\rho}_{m+j}(H) f\right)
\end{aligned}
$$

$\cdot(x)$. 
Thus

$$
\begin{aligned}
& \left|\sum_{m=-\infty}^{K} 2^{\alpha j}\left(\varphi_{j}(H) \rho_{m+j}(H) f\right)(x)\right| \\
& \leq \sum_{m=-\infty}^{K} 2^{\alpha(j+m)} 2^{\left(\alpha_{1}-\alpha\right) m} \sum_{k}\left|\Delta^{l} \frac{\varphi\left(2^{-j}(2 k+d)\right)}{\left|2^{-j}(2 k+d)\right|^{\alpha_{1}}}\right| \\
& \cdot\left|A_{k}^{l-1}\right|\left|S_{k}^{l-1}\left(\widetilde{\rho}_{m+j}(H) f\right)(x)\right| \\
& \leq C \sum_{m=-\infty}^{K} 2^{\alpha(j+m)} 2^{\left(\alpha_{1}-\alpha\right) m} M\left(\tilde{\rho}_{m+j}(H) f\right)(x),
\end{aligned}
$$

where $j \in \mathbb{Z}$ and $l-1>(9 d-8) / 6$. In addition, following the same estimate procedure of (27), we have

$$
\sum_{k}\left|\Delta^{l} \frac{\varphi\left(2^{-j}(2 k+d)\right)}{\left|2^{-j}(2 k+d)\right|^{\alpha_{1}}}\right|\left|A_{k}^{l-1}\right| \leq C .
$$

We use $\varphi(t x), 2^{-j} \leq t \leq 2^{-j+1}$, instead of $\varphi_{j}(x)=\varphi\left(2^{-j} x\right)$ in (34); this gives

$$
\begin{aligned}
& \sup _{2^{-j} \leq t \leq 2^{-j+1}}\left|\sum_{m=-\infty}^{K} 2^{\alpha j}\left(\varphi(t H) \rho_{m+j}(H) f\right)(x)\right| \\
& \leq C \sum_{m=-\infty}^{K} 2^{\alpha(j+m)} 2^{\left(\alpha_{1}-\alpha\right) m} M\left(\tilde{\rho}_{m+j}(H) f\right)(x) .
\end{aligned}
$$

For the above inequality, we first apply the $l_{q}$-quasi-norm with respect to $j$ and then apply $L^{p}$-quasi-norm with respect to $x$. Note that $\alpha_{1}>\alpha$. Then, we have

$$
\begin{aligned}
& \left\|\left(\sum_{j=-\infty}^{\infty} \sum_{2^{-j} \leq t \leq 2^{-j+1}} \mid \sum_{m=-\infty}^{K} 2^{\alpha j}\left(\varphi(t H) \rho_{m+j}(H) f\right)(x) \|^{q}\right)^{1 / q}\right\|_{L^{p}} \\
& \leq C\left\|\left(\sum_{j=-\infty}^{\infty}\left|\sum_{m=-\infty}^{K} 2^{\alpha(j+m)} 2^{\left(\alpha_{1}-\alpha\right) m} M\left(\tilde{\rho}_{m+j}(H) f\right)(x)\right|^{q}\right)^{1 / q}\right\|_{L^{p}} \\
& \leq C\left\|\left(\sum_{i=-\infty}^{\infty}\left|2^{i \alpha} M\left(\widetilde{\rho}_{i}(H) f\right)(x)\right|^{q}\right)^{1 / q}\right\|_{L^{p}} \\
& \leq C\left\|\left(\sum_{i=-\infty}^{\infty}\left|2^{i \alpha} \widetilde{\rho}_{i}(H) f(x)\right|^{q}\right)^{1 / q}\right\| \|_{L^{p}} \\
& \leq C\left\|\left(\left.\left.\sum_{i=-\infty}^{\infty}\left|2^{i \alpha} \sum_{k}\right| 2^{-i}(2 k+d)\right|^{\alpha_{1}} \rho_{i}(2 k+d) P_{k} f(x)\right|^{q}\right)^{1 / q}\right\|_{L^{p}} .
\end{aligned}
$$

Let $\psi \in S$, supp $\psi \subset \operatorname{supp} \rho$, and $\psi(x)=1$ if $x \in \operatorname{supp} \rho$. Then we have

$$
\begin{aligned}
& \left\|\left(\left.\left.\sum_{i=-\infty}^{\infty}\left|2^{i \alpha} \sum_{k}\right| 2^{-i}(2 k+d)\right|^{\alpha_{1}} \rho_{i}(2 k+d) P_{k} f(x)\right|^{q}\right)^{1 / q}\right\|_{L^{p}} \\
& =\left\|\left(\left.\left.\sum_{i=-\infty}^{\infty}\left|2^{i \alpha} \sum_{k}\right| 2^{-i}(2 k+d)\right|^{\alpha_{1}} \psi\left(2^{-i}(2 k+d)\right) \rho_{i}(2 k+d) P_{k} f(x)\right|^{q}\right)^{1 / q}\right\|_{L^{p}} \\
& =\left\|\left(\left.\left.\sum_{i=-\infty}^{\infty}\left|2^{i \alpha} \sum_{k} \Delta^{l}\right| 2^{-i}(2 k+d)\right|^{\alpha_{1}} \psi\left(2^{-i}(2 k+d)\right) A_{k}^{l-1} S_{k}^{l-1}\left(\rho_{i}(H) f\right)(x)\right|^{q}\right)^{1 / q}\right\|_{L^{p}} \\
& \leq C\left\|\left(\sum_{i=-\infty}^{\infty} 2^{i \alpha q}\left|\rho_{i}(H) f(x)\right|^{q}\right)^{1 / q}\right\|_{L^{p}}=C\|f\|_{F_{p}^{\alpha, q} .}
\end{aligned}
$$

In the proof of the above inequality, we use the following fact:

$$
\begin{gathered}
\left.\sum_{k}\left|\Delta^{l}\right| 2^{-i}(2 k+d)\right|^{\alpha_{1}} \psi\left(2^{-i}(2 k+d)\right) A_{k}^{l-1} \mid \\
\leq C \sum_{k} \mid 2^{(-i+1) l} \sum_{s=0}^{l} C_{l}^{s}\left(\left|2^{-i}(2 k+d)\right|^{\alpha_{1}}\right)^{(l-s)} \\
\cdot \psi^{(s)}\left(2^{-j}(2 k+d)\right) \mid k^{l-1} \leq C \sum_{k} 2^{2(-i+1) l}
\end{gathered}
$$

Taking $a-b=2 l>l-1>0$ yields

$$
\left.\sum_{k}\left|\Delta^{l}\right| 2^{-i}(2 k+d)\right|^{\alpha_{1}} \psi\left(2^{-i}(2 k+d)\right) A_{k}^{l-1} \mid \leq C .
$$


Let us estimate the second sum in (31) now. Similar to the estimate of the first sum, we introduce

$$
\rho_{j}^{\prime}(x)=\left|2^{-j} x\right|^{\alpha_{0}} \rho_{j}(x) \quad j \in \mathbb{Z}, \alpha_{0}<\alpha .
$$

Then we have

$$
\begin{aligned}
& \left|\sum_{m=K+1}^{\infty} 2^{\alpha j}\left(\varphi_{j}(H) \rho_{m+j}(H) f\right)(x)\right| \\
& =\mid \sum_{m=K+1}^{\infty} 2^{\alpha(j+m)} 2^{\left(\alpha_{0}-\alpha\right) m} \\
& \cdot \sum_{k} \frac{\varphi\left(2^{-j}(2 k+d)\right)}{\left|2^{-j}(2 k+d)\right|^{\alpha_{0}}} \rho_{m+j}^{\prime}(2 k+d) P_{k} f(x) \mid \\
& \leq \sum_{m=K+1}^{\infty} 2^{\alpha(j+m)} 2^{\left(\alpha_{0}-\alpha\right) m} \sum_{k}\left|\Delta^{l} \frac{\varphi\left(2^{-j}(2 k+d)\right)}{\left|2^{-j}(2 k+d)\right|^{\alpha_{0}}}\right| \\
& \cdot\left|A_{k}^{l-1}\right|\left|S_{k}^{l-1}\left(\rho_{m+j}^{\prime}(H) f\right)(x)\right| \\
& \leq C \sum_{m=K+1}^{\infty} 2^{\alpha(j+m)} 2^{\left(\alpha_{0}-\alpha\right) m} M\left(\rho_{m+j}^{\prime}(H) f\right)(x),
\end{aligned}
$$

where $j \in \mathbb{Z}$. Following the same estimate procedure of (27) gives

$$
\sum_{k}\left|\Delta^{l} \frac{\varphi\left(2^{-j}(2 k+d)\right)}{\left|2^{-j}(2 k+d)\right|^{\alpha_{0}}}\right|\left|A_{k}^{l-1}\right| \leq C .
$$

Similarly, we use $\varphi(t x), 2^{-j} \leq t \leq 2^{-j+1}$, instead of $\varphi_{j}(x)=$ $\varphi\left(2^{-j} x\right)$ in (42). Then, we have

$$
\begin{aligned}
& \sup _{2^{-j} \leq t \leq 2^{-j+1}}\left|\sum_{m=K+1}^{\infty} 2^{\alpha j}\left(\varphi(t H) \rho_{m+j}(H) f\right)(x)\right| \\
& \leq C \sum_{m=K+1}^{\infty} 2^{\alpha(j+m)} 2^{\left(\alpha_{0}-\alpha\right) m} M\left(\tilde{\rho}_{m+j}(H) f\right)(x) .
\end{aligned}
$$

Since $\alpha_{0}<\alpha$, similar to the estimate of the first sum and the estimate of (38), we arrive at

$$
\begin{aligned}
& \left\|\left(\left.\sum_{m=K+1}^{\infty}\right|_{2^{-j} \leq t \leq 2^{-j+1}} \mid \sum_{m=K+1}^{\infty} 2^{\alpha j}\left(\varphi(t H) \rho_{m+j}(H) f\right)(x) \|^{q}\right)^{1 / q}\right\|_{L^{p}} \\
& \leq C\left\|\left(\left.\left.\sum_{i=-\infty}^{\infty}\left|2^{i \alpha} \sum_{k}\right| 2^{-i}(2 k+d)\right|^{\alpha_{0}} \rho_{i}(2 k+d) P_{k} f(x)\right|^{q}\right)^{1 / q}\right\|_{L^{p}} \\
& \leq C\left\|\left(\sum_{i=-\infty}^{\infty} 2^{i \alpha q}\left|\rho_{i}(H) f(x)\right|^{q}\right)^{1 / q}\right\|_{L^{p}}=C\|f\|_{F_{p}^{\alpha, q}},
\end{aligned}
$$

which is even stronger than the desires estimate.

The proof is complete. by

The Poisson semigroup $\left\{P_{t}\right\}_{t>0}$, associated with $H$, is given

$$
P_{t} f=e^{-t H^{1 / 2}} f=\sum_{k=0}^{\infty} e^{-t(2 k+d)^{1 / 2}} P_{k} f, \quad f \in L^{2} .
$$

We refer the reader to Thangavelu's monograph [3] for a detailed description of the Poisson semigroup $\left\{P_{t}\right\}_{t>0}$ associated with $H$. In [9], the authors studied the $g$-function associated with the Poisson semigroup:

$$
g(f)(x)=\left(\int_{0}^{\infty}|\partial t f(t, x)|^{2} t d t\right)^{1 / 2},
$$

where $f \in L^{p}, 1 \leq p<\infty$, and $f(t, x)$ is the Poisson integral of $f$ that is given by the convergent series

$$
f(t, x)=\sum_{k=0}^{\infty} e^{-t(2 k+d)^{1 / 2}} \sum_{|\alpha|=k}\left\langle f, h_{\alpha}\right\rangle h_{\alpha}(x) .
$$

They obtained that, for $1<p<\infty$ and $\omega \in A_{p}$,

$$
\|g(f)\|_{L_{\omega}^{p}} \leq C\|f\|_{L_{\omega}^{p}}, \quad f \in L_{\omega}^{p},
$$

which implies

$$
C_{p}^{-1}\|f\|_{L^{p}} \leq\|g(f)\|_{L^{p}} \leq C_{p}\|f\|_{L^{p}}, \quad 1<p<\infty .
$$

That is a consequent of a general result for symmetric contraction semigroups. This general result is a refinement done by Coifman et al. [10], of the Littlewood-Paley theory for symmetric contraction semigroups satisfying, also, positivity and conservation properties which was developed by Stein [11]; see also [12]. Inspired by these results, we give the harmonic and thermic characterization of the Triebel-Lizorkin spaces associated with the Hermite expansions by the Poisson semigroup related to the Hermite expansion, which shows $F_{p}^{0,2}=L^{p}$, for $1<p<\infty$.

Theorem 5. Let $\alpha \in \mathbb{R}, 1<p<\infty$, and $1<q \leq \infty$. If $m$ is a sufficiently large natural number, then

$$
\left\|\left(\int_{0}^{\infty} t^{(m-\alpha) q}\left|\frac{\partial^{m}}{\partial t^{m}} P_{t} f(x)\right|^{q} \frac{d t}{t}\right)^{1 / q}\right\|_{L^{P}\left(\mathbb{R}^{d}\right)}
$$

(with usual modification when $q=\infty$ ) is equivalent quasinorm in $F_{p}^{\alpha, q}$.

Proof. We use Theorem 1 to prove that (51) is an equivalent quasi-norm in $F_{p}^{\alpha, q}$. Let $\varphi(x)=-|x|^{m / 2} e^{-|x|^{1 / 2}}$. Obviously, $\varphi \epsilon$ $S$; we get

$$
\begin{aligned}
\varphi\left(t^{2} H\right) f(x) & =-t^{m} H^{m / 2} e^{-t H^{1 / 2}} f(x) \\
& =t^{m} \frac{\partial^{m}}{\partial t^{m}} P_{t} f(x)
\end{aligned}
$$

It means that (51) is an equivalent quasi-norm in $F_{p}^{\alpha, q}$. Here, we substituted $t$ by $t^{2}$.

\section{Endpoint Estimates}

From Theorem 5, we know that $F_{p}^{0,2}=L^{p}$, for $1<p<\infty$. However, this conclusion does not hold when $p=1$. So, the purpose of this section is to discuss the characterization of the space $F_{1}^{0,2}$. We first present the definition of the local Hardy space below. 
Definition 6. Let $\varphi \in S\left(\mathbb{R}^{d}\right), \varphi(0)=1$, and $\varphi_{t}(x)=\varphi(t x)$. The local Hardy space associated with the Hermite operator is defined by the quasi-norm

$$
\|f\|_{h_{n}^{1}}=\left\|\sup _{0<t<2^{-n}}|\varphi(t H) f(\cdot)|\right\|_{L^{1}\left(\mathbb{R}^{d}\right)} .
$$

We now give some properties about the local Hardy space.

Proposition 7. Let $m(x)$ be an infinitely differentiable complex-valued function in $\mathbb{R}^{d}$ such that

$$
\sup _{x \in \mathbb{R}^{d}}(1+|x|)^{|\alpha|}\left|D^{\alpha} m(x)\right|<\infty
$$

for every multi-index $\alpha$. If $p=1$, then there exists a constant $C$ such that

$$
\|m(H) f(\cdot)\|_{h_{n}^{1}\left(\mathbb{R}^{d}\right)} \leq C\|f\|_{h_{n}^{1}\left(\mathbb{R}^{d}\right)}
$$

holds for all $f \in h_{n}^{1}\left(\mathbb{R}^{d}\right)$.

In order to prove Proposition 7, we recall some results from the theory of local Hardy spaces [13]; compare also [14]. A function $a$ is an atom for the local Hardy space $h_{n}^{1}$ if there is a ball $B\left(x_{0}, r\right), r \leq 2^{1-n / 2}$, such that

$$
\begin{aligned}
\operatorname{supp} a & \subset B\left(x_{0}, r\right), \\
\|a\|_{L^{\infty}} & \leq\left|B\left(x_{0}, r\right)\right|^{-1}, \\
\text { if } r & \leq 2^{-1-n / 2}, \text { then } \int a(x) d x=0 .
\end{aligned}
$$

The atomic norm in $h_{n}^{1}$ is defined by

$$
\|f\|_{h_{a, n}^{1}}=\inf \left(\sum_{j}\left|c_{j}\right|\right)
$$

where the infimum is taken over all decompositions $f=$ $\sum c_{j} a_{j} ; a_{j}$ are $h_{n}^{1}$ atoms.

Theorem GO. The norms $\|\cdot\|_{h_{n}^{1}}$ and $\|\cdot\|_{h_{a, n}^{1}}$ are equivalent with constants independent of $n \in \mathbb{Z}$.

Moreover, if $f \in h_{n}^{1}$, supp $f \subset B\left(x, 2^{1-n / 2}\right)$, then there are $h_{n}^{1}$ atoms $a_{j}$ such that supp $a_{j} \in B\left(x, 2^{2-n / 2}\right)$ and

$$
f=\sum_{j} c_{j} a_{j}, \quad \sum_{j}\left|c_{j}\right| \leq\|f\|_{h_{n}^{1}}
$$

with a constant $C$ independent of $n$.

Proof of Proposition 7. We only need to show that there exists a constant $C>0$ such that

$$
\int_{\mathbb{R}^{d}} \sup _{0<t \leq 2^{-n}}\left|\varphi_{t}(H) m(H) a(x)\right| d x \leq C,
$$

where $a$ is any $h_{n}^{1}$ atom associated with a ball $B\left(x_{0}, r\right)$. Let $B^{*}=$ $B^{*}\left(x_{0}, 2 r\right)$. Clearly, this ball is concentric with $B$. Then

$$
\begin{aligned}
\int_{B^{*}} \sup _{0<t \leq 2^{-n}}\left|\varphi_{t}(H) m(H) a(x)\right| d x & \leq C\left|B^{*}\right|^{1 / 2}\left(\int_{B^{*}} \sup _{0<t \leq 2^{-n}}\left|\sum_{k} \varphi(t(2 k+d)) m(2 k+d) P_{k} a(x)\right|^{2} d x\right)^{1 / 2} \\
& \leq C\left|B^{*}\right|^{1 / 2}\left(\int_{B^{*}} \sup _{0<t \leq 2^{-n}}\left|\sum_{k} \Delta^{l}(\varphi(t(2 k+d)) m(2 k+d)) A_{k}^{l-1} S_{k}^{l-1}(a)(x)\right|^{2} d x\right)^{1 / 2} \\
& \leq C\left|B^{*}\right|^{1 / 2}\left(\int_{B^{*}}|M(a)(x)|^{2} d x\right)^{1 / 2} \leq C\left|B^{*}\right|^{1 / 2}\left(\int_{B}|a(x)|^{2} d x\right)^{1 / 2} \\
& \leq C\left|B^{*}\right|^{1 / 2}|B|^{1 / 2}|B|^{-1} \leq C .
\end{aligned}
$$

In the above inequality, we use the fact that

$$
\sup _{0<t \leq 2^{-n}}\left|\sum_{k} \Delta^{l}(\varphi(t(2 k+d)) m(2 k+d)) A_{k}^{l-1}\right| \leq C .
$$

If $x \notin B^{*}$, we use the representation

$$
\varphi_{t}(H) m(H) a(x)=\int K_{t}(x, y) a(y) d y
$$

where

$$
\begin{aligned}
& K_{t}(x, y) \\
& \quad=\sum_{k} \varphi(t(2 k+d)) m(2 k+d) \Phi_{k}(x, y) \\
& \quad=\sum_{k} \Delta^{l}(\varphi(t(2 k+d)) m(2 k+d)) A_{k}^{l-1} S_{k}^{l-1}(x, y),
\end{aligned}
$$


and $\Phi_{k}(x, y)$ is the kernel of the projection operator $P_{k}$; see [3] for detailed description. From Lemma 3, we conclude that

$$
\begin{aligned}
& \left|K_{t}(x, y)\right| \\
& \quad \leq C \sum_{k} \frac{1}{(1+t|2 k+d|)^{a}} \frac{1}{(1+|2 k+d|)^{b}} k^{l-1} k^{d / 2} \\
& \quad \cdot\left\{\frac{1}{(1+|x-y|)^{l-(3 d-2) / 6}}\right. \\
& \left.\quad+\frac{1}{(1+|x+y|)^{l-(3 d-2) / 6}}\right\} .
\end{aligned}
$$

We choose $l-1>(3 d-2) / 6$; then we have

$$
\begin{aligned}
& \int_{\left(B^{*}\right)^{C}} \sup _{0<t \leq 2^{-n}}\left|\varphi_{t}(H) m(H) a(x)\right| d x \\
& \quad \leq C \int_{\left(B^{*}\right)^{C}} \sup _{0<t \leq 2^{-n}} \int\left|K_{t}(x, y)\right||a(y)| d y d x \\
& \quad \leq C \int_{\left(B^{*}\right)^{C}} \int\left\{\frac{1}{(1+|x-y|)^{l-(3 d-2) / 6}}\right. \\
& +\frac{1}{\left.(1+|x+y|)^{l-(3 d-2) / 6}\right\}|a(y)| d y d x} \\
& \quad \leq C \int \frac{1}{(1+|x|)^{l-(3 d-2) / 6}} d x \leq C .
\end{aligned}
$$

Then we complete our proof of Proposition 7.

If $\eta$ has the same properties as the function $\varphi$ (including $\left.\eta_{t}(x)=\eta(t x)\right)$, then the $l^{2}$-valued counterpart of $\|f\|_{h_{n}^{1}}^{\eta}$ is given by

$$
\begin{aligned}
& \left\|f_{j}\right\|_{h_{n}^{1}\left(\mathbb{R}^{d}, l^{2}\right)}^{\eta} \\
& =\left\|\sup _{0<t \leq 2^{-n}}\left(\sum_{j=-\infty}^{\infty}\left|\eta_{t}(H) f_{j}(\cdot)\right|^{2}\right)^{1 / 2}\right\|_{L^{1}\left(\mathbb{R}^{d}\right)} .
\end{aligned}
$$

In the sequel we omit the index $\eta$ in (66). Then the $l^{2}$-valued counterpart of (54), (55) reads as follows.

Proposition 8. Let $\left\{m_{j, l}(x)\right\}_{j, l=-\infty}^{\infty}$ be a sequence of infinitely differentiable complex-valued functions in $\mathbb{R}^{d}$ such that

$$
\sup _{x \in \mathbb{R}^{d}}(1+|x|)^{|\alpha|}\left(\sum_{j, l=0}^{\infty}\left|D^{\alpha} m_{j, l}(x)\right|^{2}\right)^{1 / 2}<\infty
$$

for every multi-index $\alpha$. Then there exists a constant $C$ such that

$$
\left\|\sum_{l=-\infty}^{\infty} m_{j, l}(H) f_{l}(\cdot)\right\|_{h_{n}^{1}\left(\mathbb{R}^{d}, l^{2}\right)} \leq C\left\|f_{j}\right\|_{h_{n}^{1}\left(\mathbb{R}^{d}, l^{2}\right)} .
$$

After giving the above theoretical preparations, we now state our next result.

Theorem 9. Let $\alpha=0, q=2$, and $p=1$. Then

$$
F_{1}^{0,2}\left(\mathbb{R}^{d}\right)=h_{n}^{1}\left(\mathbb{R}^{d}\right)
$$

which are equivalent quasi-norms.

Proof. Consider the following.

Step 1. The functions $f(x) \in S\left(\mathbb{R}^{d}\right)$ with compact support are dense in $F_{1}^{0,2}\left(\mathbb{R}^{d}\right)$ and dense in $h_{n}^{1}\left(\mathbb{R}^{d}\right)$; compare [13], so we assume $f(x) \in S\left(\mathbb{R}^{d}\right)$. First, we prove that

$$
\|f\|_{h_{n}^{1}\left(\mathbb{R}^{d}\right)} \sim\left\|\varphi_{j}(H) f\right\|_{h_{n}^{1}\left(\mathbb{R}^{d}, l^{2}\right)}
$$

with $\varphi_{j} \in\left\{\varphi_{j}(x)\right\}_{-\infty}^{\infty}$ (equivalent quasi-norms).

Let $f \in L^{p}\left(\mathbb{R}^{d}\right), \varphi_{j} \in\left\{\varphi_{j}(x)\right\}_{-\infty}^{\infty}$; and let $m_{j, 0}=\varphi_{j}$ and $m_{j, l}=0$ for $j, l \in \mathbb{Z}, l \neq 0$. Substituting $f_{0}=0$ and $f_{l}=0$ $(l \in \mathbb{Z}, l \neq 0)$ into (68) yields

$$
\begin{aligned}
\left\|\varphi_{j}(H) f\right\|_{h_{n}^{1}\left(\mathbb{R}^{d}, l^{2}\right)} & =\left\|\sum_{l=-\infty}^{\infty} m_{j, l}(H) f_{l}(\cdot)\right\|_{h_{n}^{1}\left(\mathbb{R}^{d}, l^{2}\right)} \\
& \leq C\left\|f_{j}\right\|_{h_{n}^{1}\left(\mathbb{R}^{d}, l^{2}\right)}=\|f\|_{h_{n}^{1}\left(\mathbb{R}^{d}\right)} .
\end{aligned}
$$

Hence $f \in h_{n}^{1}\left(\mathbb{R}^{d}, l^{2}\right)$. In order to prove the converse assertion, we assume that $f \in h_{n}^{1}\left(\mathbb{R}^{d}, l^{2}\right)$. Let

$$
\psi_{j}(x)=\sum_{r=-1}^{1} \varphi_{j+r}(x) \quad\left(\text { with } \varphi_{-1}=0\right) .
$$


If $x \in \operatorname{supp} \varphi_{j}$, it is easy to get $\psi_{j}=1$. Let $m_{0, l}=\psi_{l}$ and $m_{j, l}=0$ if $j \in \mathbb{Z}, l \in \mathbb{Z}$, and $l \neq 0$. We apply (68) with $f_{j}=\varphi_{j}(H) f$. Then

$$
\begin{aligned}
& \left\|\sum_{l=-\infty}^{\infty} m_{j, l}(H) f_{l}(\cdot)\right\|_{h_{n}^{1}\left(\mathbb{R}^{d}, l^{2}\right)} \\
& =\left\|\sup _{0<t \leq 2^{-n}}\left(\sum_{j=-\infty}^{\infty}\left|\eta_{t}(H) \sum_{l=-\infty}^{\infty} m_{j, l}(H) f_{l}(\cdot)\right|^{2}\right)^{1 / 2}\right\|_{L^{1}\left(\mathbb{R}^{d}\right)} \\
& =\left\|\sup _{0<t \leq 2^{-n}}\left|\eta_{t}(H) \sum_{l=-\infty}^{\infty} \psi_{l}(H) f_{l}(\cdot)\right|\right\|_{L^{1}\left(\mathbb{R}^{d}\right)} \\
& =\left\|\sup _{0<t \leq 2^{-n}} \mid \eta_{t}(H) \sum_{l=-\infty}^{\infty} \varphi_{l}(H) f(\cdot)\right\| \|_{L^{1}\left(\mathbb{R}^{d}\right)} \\
& =\left\|\sup _{0<t \leq 2^{-n}}\left|\eta_{t}(H) f(\cdot)\right|\right\|_{L^{1}\left(\mathbb{R}^{d}\right)} \leq C\left\|f_{j}\right\|_{h_{n}^{1}\left(\mathbb{R}^{d}, l^{2}\right)} \\
& =\left\|\varphi_{j}(H) f\right\|_{h_{n}^{1}\left(\mathbb{R}^{d}, l^{2}\right)} \cdot
\end{aligned}
$$

Therefore

$$
\|f\|_{h_{n}^{1}\left(\mathbb{R}^{d}\right)} \leq C\left\|\varphi_{j}(H) f\right\|_{h_{n}^{1}\left(\mathbb{R}^{d}, l^{2}\right)} .
$$

Thus, (70) holds.

Step 2. We recall that $\eta(0)=1$. It follows from (66) with $f_{j}=$ $\varphi_{j}(H) f$ and $(70)$ that

$$
\begin{aligned}
& \left\|f_{j}\right\|_{h_{n}^{1}\left(\mathbb{R}^{d}, l^{2}\right)} \\
& =\left\|\varphi_{j}(H) f\right\|_{h_{n}^{1}\left(\mathbb{R}^{d}, l^{2}\right)} \\
& =\left\|\sup _{0<t \leq 2^{-n}}\left(\sum_{j=-\infty}^{\infty}\left|\eta_{t}(H) \varphi_{j}(H) f(\cdot)\right|^{2}\right)^{1 / 2}\right\|_{L^{1}\left(\mathbb{R}^{d}\right)} \\
& \geq C\left\|\left(\sum_{j=-\infty}^{\infty}\left|\varphi_{j}(H) f(\cdot)\right|^{2}\right)^{1 / 2}\right\|_{L^{1}\left(\mathbb{R}^{d}\right)} \\
& =\|f\|_{F_{1}^{0,2}\left(\mathbb{R}^{d}\right)} .
\end{aligned}
$$

Step 3. We prove the converse assertion of (75). From (66) and (75) we have

$$
\begin{aligned}
& \|f\|_{h_{n}^{1}\left(\mathbb{R}^{d}\right)} \\
& \leq C\left\|\sup _{0<t \leq 2^{-n}}\left(\sum_{j=-\infty}^{\infty}\left|\eta_{t}(H) \varphi_{j}(H) f(\cdot)\right|^{2}\right)^{1 / 2}\right\|_{L^{1}\left(\mathbb{R}^{d}\right)} .
\end{aligned}
$$

Since $\eta(x)$ has compact support, $\eta_{t}(2 k+d) \varphi_{j}(2 k+d) \neq 0$ implies $k \approx 2^{j}$. So, we have

$$
\begin{aligned}
& \sup _{0<t \leq 2^{-n}}\left(\sum_{j=-\infty}^{\infty}\left|\eta_{t}(H) \varphi_{j}(H) f(x)\right|\right) \\
& =\sup _{0<t \leq 2^{-n}}\left(\sum_{j=-\infty}^{\infty}\left|\sum_{k} \eta(t(2 k+d)) P_{k} \varphi_{j}(H) f(x)\right|\right) \\
& \leq C \sup _{0<t \leq 2^{-n}} \int_{\mathbb{R}^{d}}\left|K_{t}(x, y) \varphi_{j}(H) f(y) d y\right|,
\end{aligned}
$$

where

$$
\begin{aligned}
& \left|K_{t}(x, y)\right|=\left|\sum_{k} \eta_{t}(2 k+d) \Phi_{k}(x, y)\right| \\
& \quad=\left|\sum_{k} \Delta^{l}\left(\eta_{t}(2 k+d)\right) A_{k}^{l-1} S_{k}^{l-1}(x, y)\right| \\
& \quad \leq C \sum_{k} \frac{1}{(1+t|2 k+d|)^{a}} k^{l-1} k^{d / 2}
\end{aligned}
$$

$$
\begin{aligned}
& \cdot\left\{\frac{1}{\left(1+2^{j / 2}|x-y|\right)^{l-(3 d-2) / 6}}\right. \\
& \left.+\frac{1}{\left(1+2^{j / 2}|x+y|\right)^{l-(3 d-2) / 6}}\right\} .
\end{aligned}
$$

If $\varphi_{j, s}^{*}(f)(x)$ is the maximal function in (11), where $s=l-$ $(3 d-2) / 6>d / \min (p, q)$, then by Theorem GS we get

$$
\begin{aligned}
\|f\|_{h_{n}^{1}\left(\mathbb{R}^{d}\right)} & \leq C\left\|\left(\sum_{j=-\infty}^{\infty}\left|\varphi_{j, s}^{*}(f)(x)\right|^{2}\right)^{1 / 2}\right\|_{L^{1}\left(\mathbb{R}^{d}\right)} \\
& \leq C\|f\|_{F_{1}^{0,2}\left(\mathbb{R}^{d}\right)} .
\end{aligned}
$$

In summary, (75) and (79) tell us that the quasi-norms $\|f\|_{h_{n}^{1}\left(\mathbb{R}^{d}\right)}$ and $\|f\|_{F_{1}^{0,2}\left(\mathbb{R}^{d}\right)}$ are equivalent on the functions $f \in S\left(\mathbb{R}^{d}\right)$ with compact support. Theorem 9 follows by completion. 


\section{The Boundedness of the Riesz Transform on $F_{p}^{\alpha, q}$}

This section presents the proof of the boundedness of the Riesz transforms associated with Hermite expansions on the space $F_{p}^{\alpha, q}$.

Since

$$
L=-\frac{1}{2}[(\nabla+x) \cdot(\nabla-x)+(\nabla-x) \cdot(\nabla+x)],
$$

it is reasonable to define the Riesz transforms $R_{j}^{ \pm}, j=$ $1,2, \ldots, d$, by

$$
R_{j}^{ \pm}=\left(\frac{\partial}{\partial x_{j}} \pm x_{j}\right) L^{-1 / 2} .
$$

This definition was suggested by Thangavelu [15]; compare also [3]. The definition fits a general framework and, in dimension one, matches the notion of conjugacy for the Hermite function expansions investigated by Gosselin and Stempak [16]. In [17], Stempak and Torrea have already proved that the kernels of the Riesz transforms satisfy the Calderón-Zygmund standard conditions. Their results are as below.

Theorem KJ. The Riesz operators $R_{j}^{ \pm}$, initially defined on $L^{2}$, are Calderón-Zygmund operators associated with the (Calderón-Zygmund) kernels $R_{j}^{ \pm}(x, y)$, which satisfy

$$
\begin{array}{r}
\left|R_{j}^{ \pm}(x, y)\right| \leq \frac{C}{|x-y|^{d}}, \\
\left|\nabla_{x} R_{j}^{ \pm}(x, y)\right|+\left|\nabla_{y} R_{j}^{ \pm}(x, y)\right| \leq \frac{C}{|x-y|^{d+1}} .
\end{array}
$$

We now state the last result of this paper.

Theorem 10. Let $\alpha \in \mathbb{R}, 0<p$, and $q<\infty$. Then the Riesz transform associated with $H$ is a bounded operator on $\|f\|_{F_{p}^{\alpha, q}}$.

Proof. Using the equivalent quasi-norm of $F_{p}^{\alpha, q}$ defined in (51), we yield that

$$
T R_{j}^{ \pm}=R_{j}^{ \pm} T
$$

where $T$ is the operator $f \rightarrow T(f)$ that is defined by

$$
T(f)(x)=\left\{\frac{\partial^{m}}{\partial t^{m}} P_{t} f(x)\right\}_{t>0} .
$$

We obtain

$$
\begin{aligned}
\left\|R_{j}^{ \pm}\right\|_{F_{p}^{\alpha, q}} & =\left\|\left(\int_{0}^{\infty}\left|t^{(m-\alpha)} T R_{j}^{ \pm} f(x)\right|^{q} \frac{d t}{t}\right)^{1 / q}\right\|_{L^{p}\left(\mathbb{R}^{d}\right)} \\
& =\left\|\left(\int_{0}^{\infty}\left|t^{(m-\alpha)} R_{j}^{ \pm} T f(x)\right|^{q} \frac{d t}{t}\right)^{1 / q}\right\|_{L^{p}\left(\mathbb{R}^{d}\right)},
\end{aligned}
$$

where

$$
\begin{aligned}
& \left(\int_{0}^{\infty}\left|t^{(m-\alpha)} R_{j}^{ \pm} T f(x)\right|^{q} \frac{d t}{t}\right)^{1 / q} \\
& \left.\quad \leq \int_{\mathbb{R}^{d}}\left(\int_{0}^{\infty} \mid t^{(m-\alpha)} R_{j}^{ \pm}(x, y) T f(y)\right)^{q} t d t\right)^{1 / q} d y \\
& \quad \leq C \int_{\mathbb{R}^{d}} \frac{1}{|x-y|^{d}}\left(\int_{0}^{\infty}\left|t^{(m-\alpha)} T f(y)\right|^{q} t d t\right)^{1 / q} d y \\
& \quad \leq C\left(\int_{\mathbb{R}^{d}} \frac{1}{|x-y|^{d p^{\prime}}} d y\right)^{1 / p^{\prime}} \\
& \quad\left(\int_{\mathbb{R}^{n}}\left(\int_{0}^{\infty}\left|t^{(m-\alpha)} T f(y)\right|^{q} t d t\right)^{p / q} d y\right)^{1 / p} \\
& \quad \leq C\left(\int_{\mathbb{R}^{d}} \frac{1}{|x-y|^{d p^{\prime}}} d y\right)^{1 / p^{\prime}}\|f\|_{F_{p}^{\alpha, q} .}
\end{aligned}
$$

Applying the $L^{p}$-norm with respect to $x$ in (87) gives

$$
\left\|R_{j}^{ \pm} f\right\|_{F_{p}^{\alpha, q}} \leq C\|f\|_{F_{p}^{\alpha, q}} \text {. }
$$

The results presented in Theorem 10 can be further enlarged to the boundedness of the $g$-functions of Littlewood-Paley defined with either the heat semigroup or the Poisson semigroup. We use a theorem to describe this bounded result.

Theorem 11. If $g$ is commutative with the operator $T$ defined by (85) and if the kernel of $g$ satisfies the Calderón-Zygmund size condition, then $g$ is bounded on $F_{p}^{\alpha, q}$.

Since the proof of Theorem 11 is very similar to the proof of Theorem 10, we omit the proof here in order to make the paper concise.

\section{Conflict of Interests}

The authors declare that there is no conflict of interests regarding the publication of this paper.

\section{Acknowledgments}

The authors thank the anonymous referees cordially for their valuable suggestions on this paper. This work was partially supported by NSF of China (Grants nos. 11271175, 11271162), AMEP (DYSP) of Linyi University, and Macao Science and Technology Development Fund, MSAR (Ref. 018/2014/A1).

\section{References}

[1] H. Triebel, Theory of Function Spaces II, vol. 84 of Monographs in Mathematics, Birkhäuser, Basel, Switzerland, 1992. 
[2] W. Yuan, W. Sickel, and D. C. Yang, Morrey and Campanato meet Besov, Lizorkin and Triebel, vol. 2005 of Lecture Notes in Mathematics, Springer, Berlin, Germany, 2010.

[3] S. Thangavelu, Lectures on Hermite and Laguerre Expansions, vol. 42 of Mathematical Notes, Princeton University Press, Princeton, NJ, USA, 1993.

[4] J. Epperson, "Triebel-Lizorkin spaces for Hermite expansions," Studia Mathematica, vol. 114, no. 1, pp. 87-103, 1995.

[5] J. Dziubański, "Triebel-Lizorkin spaces associated with LAGuerre and Hermite expansions," Proceedings of the American Mathematical Society, vol. 125, no. 12, pp. 3547-3554, 1997.

[6] P. Petrushev and Y. Xu, "Decomposition of spaces of distributions induced by Hermite expansions," The Journal of Fourier Analysis and Applications, vol. 14, no. 3, pp. 372-414, 2008.

[7] G. Olafsson and S. Zheng, "Harmonic analysis related to schrödinger operators," Contemporary Mathematics, vol. 464, pp. 213-230, 2008.

[8] E. Harboure, L. de Rosa, C. Segovia, and J. L. Torrea, " $L^{P}$ dimension free boundedness for Riesz transforms associated to Hermite functions," Mathematische Annalen, vol. 328, no. 4, pp. 653-682, 2004.

[9] K. Stempak and J. L. Torrea, "On $g$-functions for Hermite function expansions," Acta Mathematica Hungarica, vol. 109, no. 1-2, pp. 99-125, 2005.

[10] R. R. Coifman, R. Rochberg, and G. Weiss, "The $L^{p}$ version of von Neumann's inequality and Littlewood-Paley-Stein theory," in Linear Spaces and Approximation, P. L. Butzer and B. S. Nagy, Eds., pp. 53-67, Birkhäuser, Basel, Switzerland, 1978.

[11] E. M. Stein, Topics in Harmonic Analysis Related to the Littlewood-Paley Theory, Annals of Mathematics Studies, Princeton University Press, Princeton, NJ, USA, 1970.

[12] S. Meda, "A general multiplier theorem," Proceedings of the American Mathematical Society, vol. 110, no. 3, pp. 639-647, 1990.

[13] D. Goldberg, "A local version of real Hardy spaces," Duke Mathematical Journal, vol. 46, no. 1, pp. 27-42, 1979.

[14] J. Dziubański and J. Zienkiewicz, "Hardy space $H^{1}$ associated to Schrödinger operator with potential satisfying reverse Hölder inequality," Revista Matemática Iberoamericana, vol. 15, no. 2, pp. 279-296, 1999.

[15] S. Thangavelu, "Riesz transforms and the wave equation for the Hermite operator," Communications in Partial Differential Equations, vol. 15, no. 8, pp. 1199-1215, 1990.

[16] J. Gosselin and K. Stempak, "Conjugate expansions for Hermite functions," Illinois Journal of Mathematics, vol. 38, no. 2, pp. 177197, 1994.

[17] K. Stempak and J. L. Torrea, "Poisson integrals and Riesz transforms for Hermite function expansions with weights," Journal of Functional Analysis, vol. 202, no. 2, pp. 443-472, 2003. 


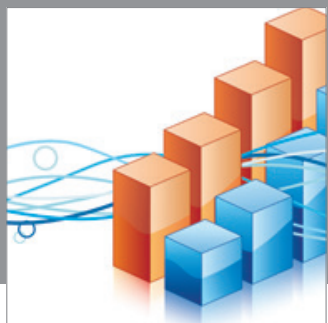

Advances in

Operations Research

mansans

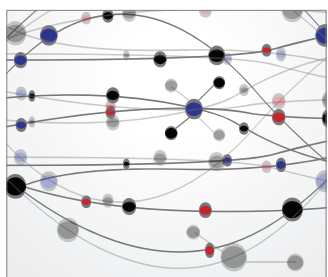

The Scientific World Journal
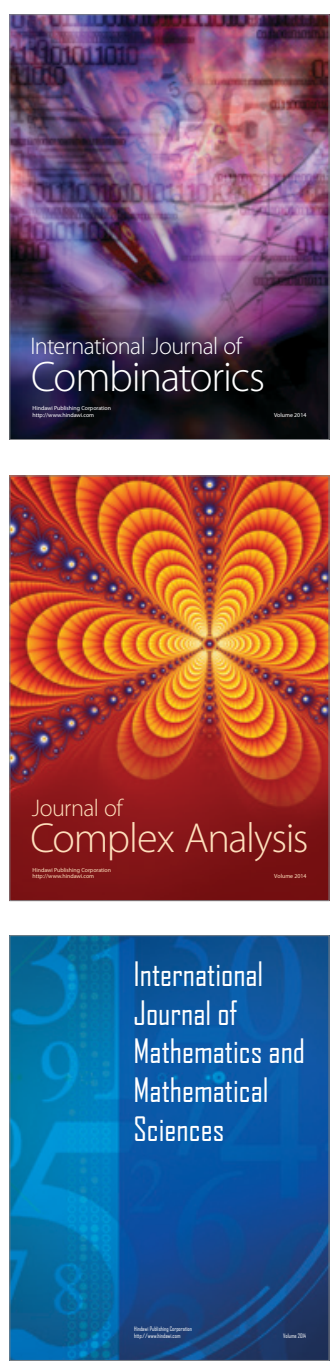
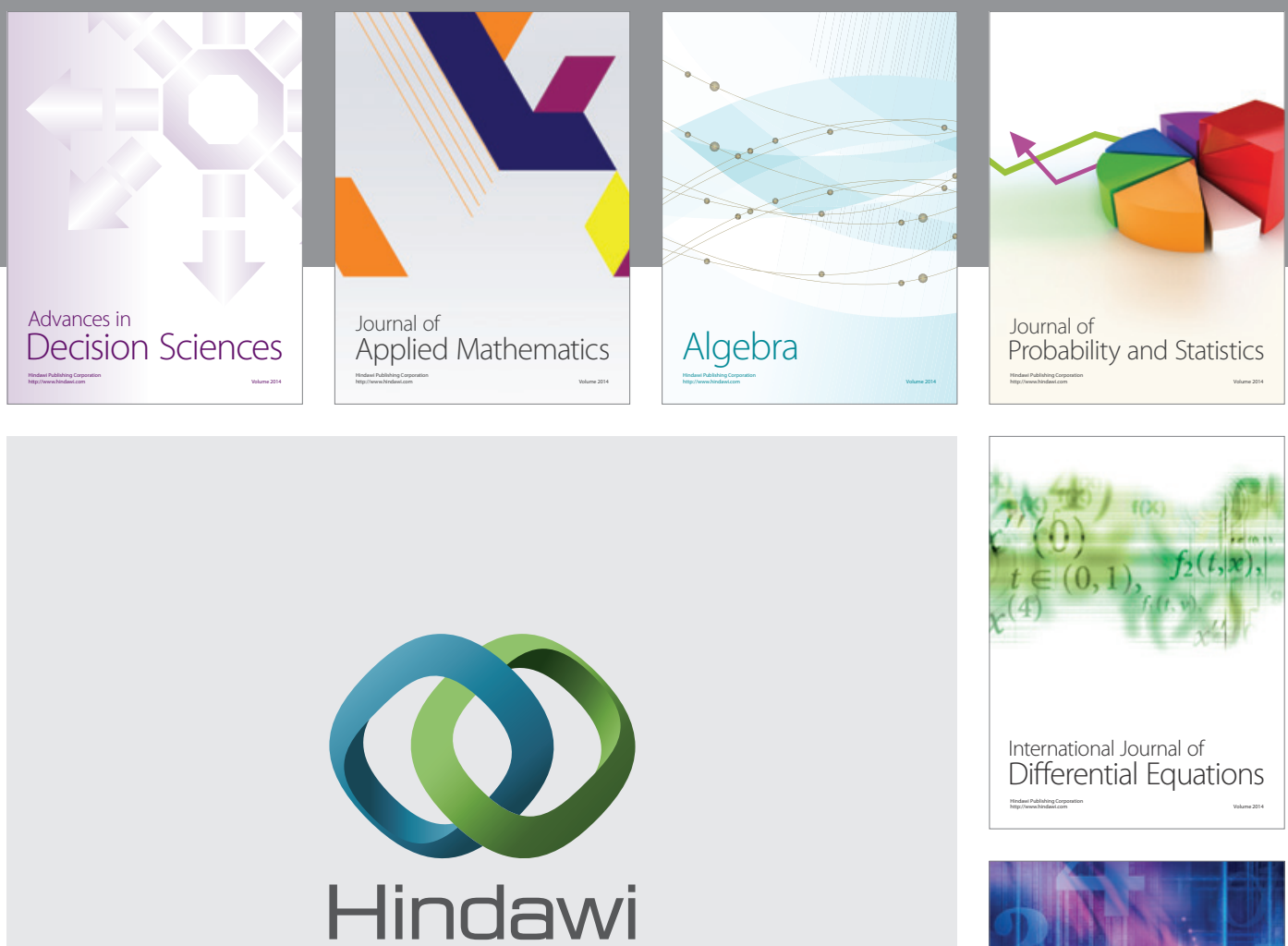

Submit your manuscripts at http://www.hindawi.com
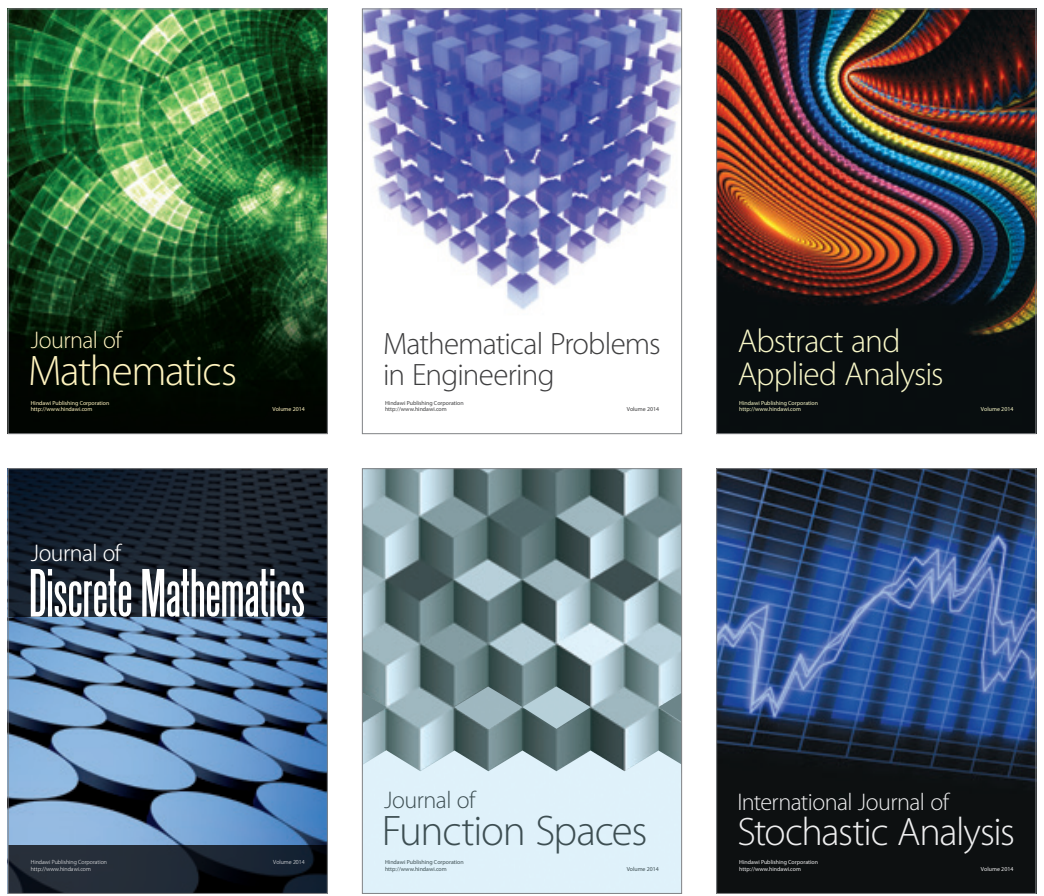

Journal of

Function Spaces

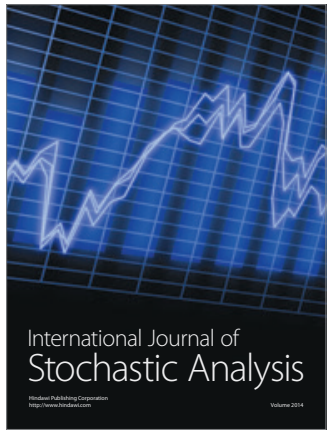

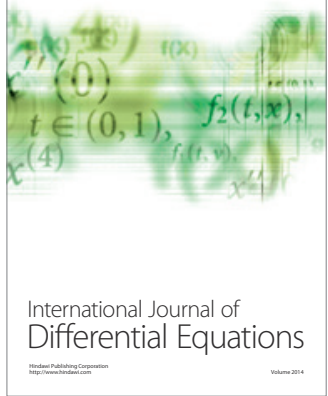
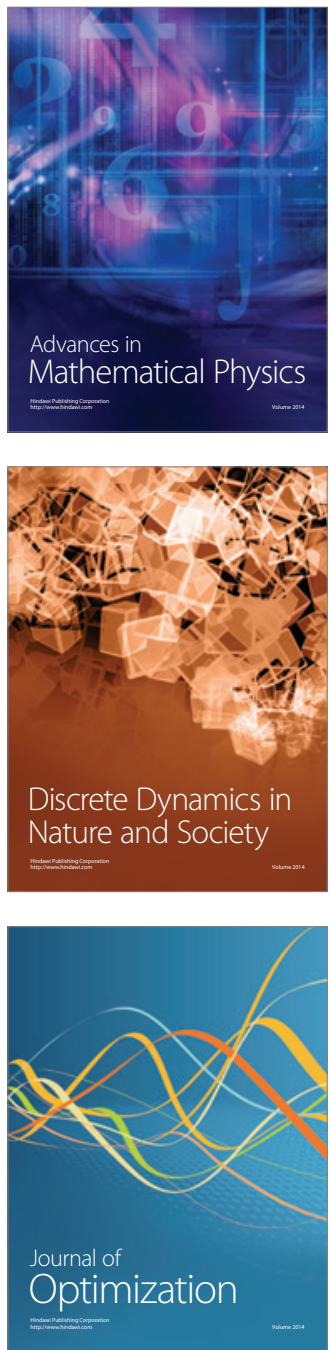